\title{
Quiet Area Tests of a Ka-band Compact Range
}

\author{
Yuxin Kou1, Xuetian Wang1 \& Chi Liu1 \\ ${ }^{1}$ School of Information and Electronics, Beijing Institute of Technology, Beijing, 100081, China
}

Keywords: quiet-area, Ka-band, compact range.

\begin{abstract}
Because of the limited size of a common microwave anechoic chamber, compact range testing methods have been developed to be a hot spot in recent years. But an ordinary compact range can not meet the need of millimeter wave (with high frequency, 200MHz 20GHz) test. So this article uses a millimeter compact range to do the test at Ka-band, and the main purpose of the test is to determine the range of compact range quiet area. Based on the test results, the quiet area of the millimeter compact range is about $400 \mathrm{~mm} * 400 \mathrm{~mm}$ at the distance of $3 \mathrm{~m}$ to the center of the reflecting surface, and the quiet area is of pretty good property.
\end{abstract}

\section{Introduction}

Because of the limited size of a common microwave anechoic chamber, it is difficult to obtain some satisfactory plane wave conditions for a large target. So we prefer a new kind of equipment which can change the spherical wave into plane wave by using a smooth reflecting surface at a short range. It is CATR (the compact antenna test range). CATR is very convenient for antenna testing, and generally speaking, it usually includes two parts: an antenna with a precision reflecting surface and a microwave anechoic chamber. And then, a quiet area is created through the perfect cooperation of the processed edge of the reflecting surface, the antenna and the microwave anechoic chamber. But one hand, an ordinary CATR can not meet the need of millimeter wave (with high frequency: $200 \mathrm{MHz}-20 \mathrm{GHz}$ ) test, on the other hand, the millimeter wave could be easily effected by weather conditions such as the falling rain or the fog, so the far-field range $R \geq \frac{2 D^{2}}{\lambda}$ (D is for the size of the antenna aperture, $\lambda$ is for antenna operating wavelength, $\mathrm{R}$ is for the minimum distance of the antenna test) testing conditions are very harsh.

In fact, the past CATR system has defects on test band, the quiet area size and test performance, so a brand new millimeter compact range is willing to come into being[1-3].

This paper describes the detection of a Ka-band quiet area in a millimeter compact range. Actually, we use the absorbing materials which is spread everywhere of the microwave anechoic chamber to make the minimum reflectance of walls, thus an approximated area of free space is expected to be detected. It is called quiet area. What's more, the amplitude in quiet is asked to smaller than $\pm 1 \mathrm{~dB}$, the phase is asked to be $\pm 10^{\circ}$.

\section{The Compact Range Theory}

The test uses the compact antenna test range system making by Xi'an Hengda Microwave Technology Development Company. It is a compact range formed by a rotating paraboloid, and the feed source is put at the focus of the paraboloid. According to the geometrical optics, all points of the antenna aperture can be considered as sub-wave source to generate spherical wave, and the enveloping surface of these sub-wave source becomes the new wavefront. Each new wavefront generated in this compact range is parallel to the CATR aperture, and the plane wave propagation rays are perpendicular to the wavefront. Then the millimeter CATR achieves the goal of a single plane wave. The scattered field will spread to all sides.

When the scattered field of different directions reaches the reflecting surface, only the single plane wave with the aperture in the vertical direction can be focused to the feed point, and received by the receiving antenna. Other waves can't be focused to the feed point. So the receiving antenna can only receive the single plane wave. So, compact range can reach the goal of the condition that 
single wave transmitting and receiving provided by RCS measurement in far field. What's more, the compact range requires that the feed point should provide a uniform radiation pattern in the illuminating angle range. We use the form of a conical horn surface with a slot horn to change the boundary conditions, and to suppress the aperture current. And then we can see the same field distribution between the $\mathrm{E}$ field and the $\mathrm{H}$ field and get the better equalization pattern, low side-lobe level and low cross-polarization in the compact range.

\section{CATR System Quiet Area Detection}

\subsection{Test Principle and Procedure.}

The testing principle of amplitude and phase in electric field of the compact range plane wave radiation field area is as shown in Fig.1.

The electromagnetic field amplitude of plane wave is constant and independent of the coordinate variables, so the magnitude of amplitude and phase are all the same at the constant phase front.

We use a two-dimensional scanning frame to do a two-dimensional scanning frame on a plane perpendicular to the electromagnetic wave propagation direction, and the receiving antenna (the center frequency is $32 \mathrm{GHz}$ ) is fixed on the two-dimensional scanning frame. And then we observe and keep a record of the S21 magnitude-phase characteristics of each receiving and transmitting channel in the vector network analyzer. If the S21 amplitude-phase characteristics can fit the plane wave conditions, that is to say the magnitude and phase are the same at the constant phase front, and we can determine that the range is plane wave.

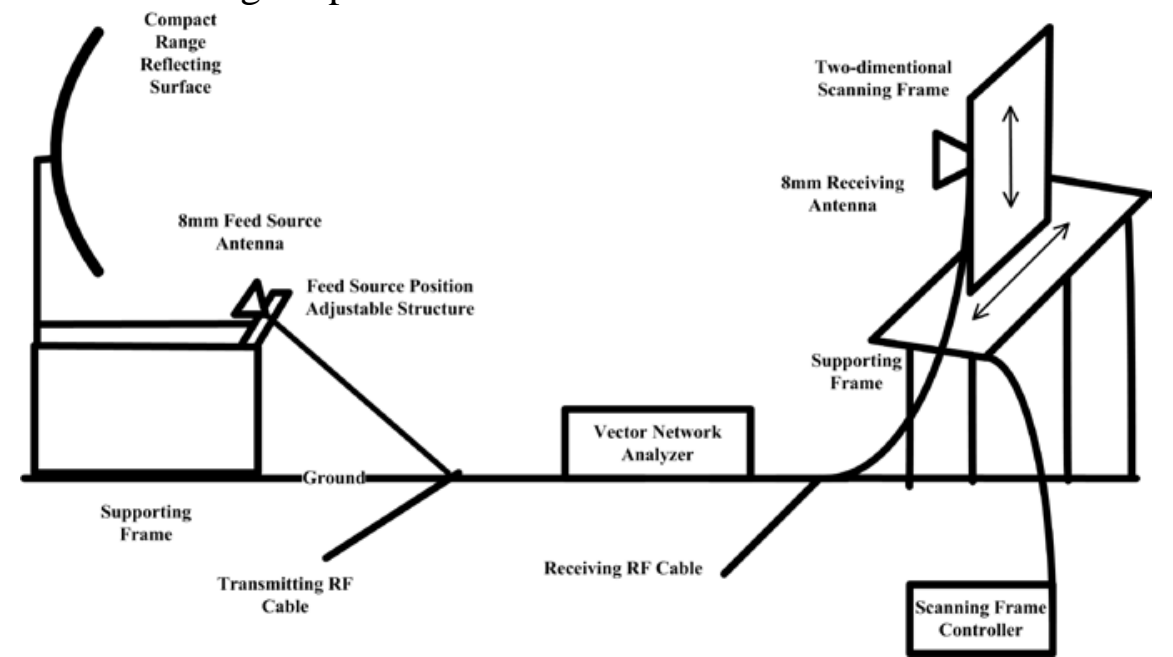

Fig.1: Testing principle of CATR system quiet area

This test is aimed at figuring out the space scope of plane wave transmission of an anechoic chamber.

The reflecting surface and the feed source antenna is shown on the left of the Fig.2., and the twodimensional scanning frame is on the right, and the two-dimensional scanning frame is used to detect the plane wave. Let the CATR reflecting surface aperture be vertically connected with the 'center to center line' of the reflecting surface and the receiving antenna. The vector network analyzer is working on $26.4 \mathrm{GHz} 40 \mathrm{GHz}$, without spread spectrum components. The distance between the CATR reflecting surface and the receiving antenna is $3 \mathrm{~m}$. The place of the horizontal and vertical dimension is focused on the center of CATR reflecting surface. The horizontal dimension is parallel to the ground, and the reflecting surface brackets are covered by the waveabsorbing material. There is no electric wave reflecting in the main lobe of feed source antenna. The transmitting and the receiving RF cables are phase stable cables. The vector network analyzer and the two-dimensional scanning frame controller are put at the right side of the main beam and covered by wave-absorbing material. The two-dimensional scanning frame starts moving at $\mathrm{X}=250 \mathrm{~mm}$ according to the vertical height of $0.1 \mathrm{~m}$ intervals from top to bottom, and then moves at 
$\mathrm{Y}=250 \mathrm{~mm}$ according to the horizontal length of $0.1 \mathrm{~m}$ intervals from left to right. And we next observe and keep the record of each S21 amplitude-phase characteristics.

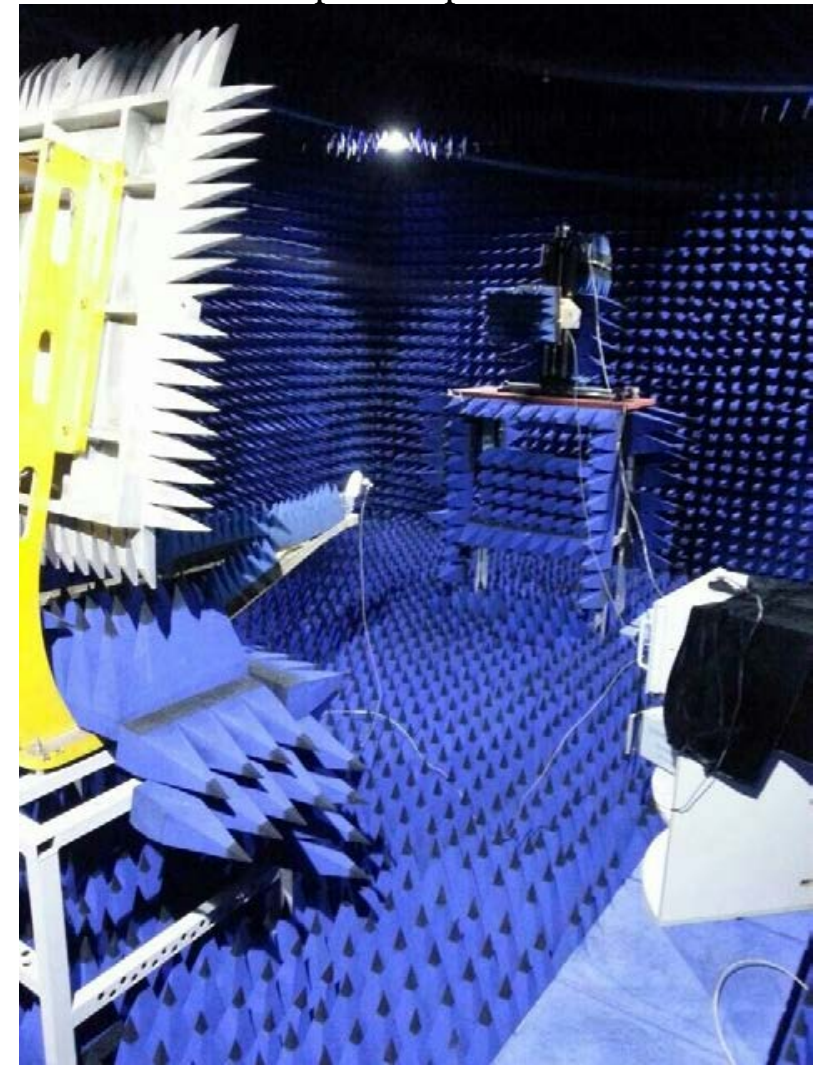

Fig.2: Testing environment of CATR system

\subsection{Test Results.}

Firstly, we fix the $\mathrm{X}$-axis at $250 \mathrm{~mm}$, and scan $\mathrm{Y}$-axis from top to bottom, observing the amplitude and phase changes of vertical dimension. The observation frequency is 32GHz. Then we fix the Y-axis at $250 \mathrm{~mm}$, and scan X-axis from left to right, observing the amplitude and phase changes of horizontal dimension. The observation frequency is still $32 \mathrm{GHz}$, and the results are as Fig.3 shown.

$\mathrm{X}$-axis is fixed at the center axis, and we scan the amplitude and phase changes of vertical dimension at y axis .The testing conditions between $50 \mathrm{~mm}-450 \mathrm{~mm}$ are as follows. The amplitude value changes between $-62.2 \mathrm{~dB} \sim 64.2 \mathrm{~dB}$, and phase value changes from $99.5^{\circ} \sim 113.3^{\circ}$.

$\mathrm{Y}$-axis is fixed at the center axis, and we scan the amplitude and phase changes of horizontal dimension at $\mathrm{x}$ axis .The testing conditions between $50 \mathrm{~mm}-450 \mathrm{~mm}$ are as follows. The amplitude value changes between $-62.3 \mathrm{~dB} \sim 64.0 \mathrm{~dB}$, and phase value changes from $102.3^{\circ} \sim 116.5^{\circ}$. 

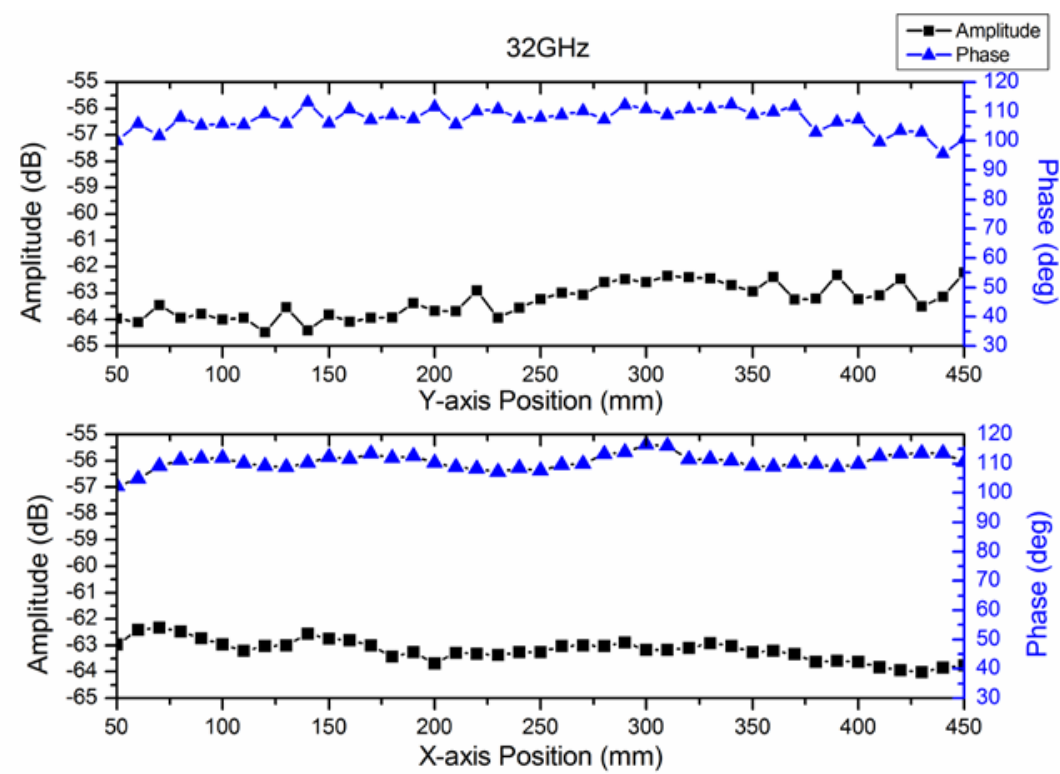

Fig.3: Results of testing on $32 \mathrm{GHz}$

And then, let the scanning frame fixed at X-axis (Y-axis) 10mm per time, scan the amplitude and phase changes of vertical dimension (horizontal dimension) at $10 \mathrm{~mm}$ per time. The changes of amplitude value of vertical dimension and horizontal dimension are all less than $\pm 0.75 \mathrm{~dB}$. Some of these changes are about $\pm 1 \mathrm{~dB}$, and the changes of phase value are less than $\pm 8^{\circ}$ in vertical dimension. The changes of phase value are about $\pm 5^{\circ}$ in horizontal dimension.

\section{Conclusions}

From the analysis of the results given above, the changes of amplitude value of vertical dimension and horizontal dimension are all less than $\pm 0.75 \mathrm{~dB}$, only a small part of the variation is about $\pm 1 \mathrm{~dB}$, and the figures above are suitable for the requirement of $\pm 1 \mathrm{~dB}$ of CATR quiet area's amplitude variation. The changes of phase value of vertical dimension are about $\pm 8^{\circ}$, and changes of phase value of horizontal dimension are about $\pm 5^{\circ}$. The figures above are suitable for the requirement of $\pm 10^{\circ}$ of CATR quiet area's phase variation. The reasons why the figures of amplitude value and phase value are not stable might be the floating phase of cables during the movement of the two-dimensional scanning frame and the mismachining tolerance of the reflecting surface. Metastable phase of cable is less than $\pm 0.5^{\circ}$, and the background noise of anechoic chamber is under $-110 \mathrm{~dB}$. So we can determine that the quiet area of this millimeter compact range is about $400 \mathrm{~mm} \times 400 \mathrm{~mm}$ at the distance of $3 \mathrm{~m}$ to the center of the reflecting surface.

The determination of CATR quiet area proves that this millimeter compact range is valuable at the frequency of $8 \mathrm{~mm}$. It makes important significance of further exploration for higher frequency test. And then we can use it to do more effective test such as the test for antenna parameters and the test for RCS.

\section{References}

[1] Zhuoran Zhang, Xian Qiu, Guoyu He. High performance single reflector compact range[C]. National Conference on Microwave and Millimeter Waves.

[2] Xiao-gang G, Sui-xue W. High Performance Single Cylindrical Reflector Compact Range Chamber[J]. Journal of China Academy of Electronics and Information Technology, 2007, pp. 653-657. 
[3] Rusch W V T, Prata A J, Yang C C, et al. A millimeter-wave compact range using an axiallysymmetric paraboloid in a Gregorian configuration[C]. Antennas and Propagation Society International Symposium, 1991. AP-S. Digest. IEEE, 1991:1186 - 1189. 